\title{
Reconfigurable Metamaterial Structure at Millimeter Wave Frequency Range
}

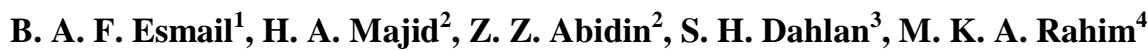 \\ ${ }^{1,2}$ Faculty of Engineering Technology, Universiti Tun Hussein Onn Malaysia, Parit Raja, Johor, Malaysia \\ ${ }^{3}$ Research Center of Applied Electromagnetics, Universiti Tun Hussein Onn Malaysia, Parit Raja, Johor, Malaysia \\ ${ }^{4}$ Faculty of Electrical Engineering, Universiti Teknologi Malaysia, Johor Bahru, Johor, Malaysia
}

\begin{abstract}
Article Info
Article history:

Received Jul 18, 2017

Revised Oct 20, 2017

Accepted Nov 14, 2017

\section{Keyword:}

$5 \mathrm{G}$

Metamaterial loss

Millimeter wave

Refractive index reconfigrable

ABSTRACT

In this paper, reconfigurable metamaterial structure at millimeter wave frequency range was designed and simulated for a future fifth generation (5G) mobile-phone beam switching applications. The new proposed structure was composed of a bridge-shaped resonator (BSR) in the front face and strip line at the back face of the unit cell which operates at $28 \mathrm{GHz}$. First, nonreconfigurable low loss BSR unit cell was designed and subsequently, the reconfigurability was achieved using four switches formed in the gaps of the structure. The proposed structure achieves the lowest loss and almost full transmission among its counterparts by $-0.06 \mathrm{~dB}$ ( 0.99 in linear scale). To demonstrate the reconfigurability of the metamaterial, the reflection and transmission coefficients and real parts of the effective refractive index at each reconfigured frequency were studied and investigated. Simulation results showed that a high transmission and reflection peaks occur at each resonance frequency according to change the state of the switches.
\end{abstract}

Copyright $\odot 2017$ Institute of Advanced Engineering and Science. All rights reserved.

\section{Corresponding Author:}

Huda Bin A Majid,

Faculty of Engineering Technology,

Universiti Tun Hussein Onn Malaysia,

86400 Parit Raja, Johor, Malaysia.

Email: mhuda@uthm.edu.my

\section{INTRODUCTION}

Metamaterials (MTM) are artificial materials that exhibit unique responses to electromagnetic wave (EM) properties such as negative refractive index, inverse Doppler shift, and reversal of Cherenkov radiation [1]. The simultaneously negative permittivity and permeability properties were proved theoretically by Victor Veselago in 1967 and verified experimentally after 29 years by Pendry in 1996 [2], [3]. Because of these unusual electromagnetic properties, metamaterials can be utilized as a part of numerous important applications such as super-lenses [4], cloaking technology [5], design and enhance the antenna performance [6]. The study of the negative index metamaterials has been enhanced through various strategies and processes. However, few issues have been encountered such as the narrow bandwidths and losses that limit the spectrum and the range of their applications. Metamaterials suffer from high losses when the frequency is pushed to the higher range such as millimeter wave (MMW) bands [7]. However, the losses are very low and the unusual electromagnetic properties of the metamaterials can still be achieved within the microwave frequency region. The losses can give negative influences and adverse effects toward the realizations of the unique electromagnetic properties of the metamaterials. Throughout the years, the researchers had been proposed various technique to reduce such losses.

A number of diverse techniques are extensively reported in the literature to compensate the metamaterials losses at microwave and terahertz such as tailoring geometry of metamaterial unit cell [8], using the electromagnetically induced transparency (EIT) at low frequency range [9], integrate an active 
material [10]. Hence, new metamaterials structures with relatively low loss are desirable. The introduction of a new structure of metamaterial with proper arrangements, can overcome the losses and improve the performance of the devices at high frequency range.

Reconfigurable Metamaterials attract more attention in recent years since they show a variable response to an incident EM wave. The EM behavior of metamaterial unit cell is modified intentionally using different reconfigurable methods such as mechanical, thermal and electrical. The metamaterial can be reconfigured mechanically by changing the distance between the metamaterial elements, or the distance between the metamaterial and the substrate, thus modifying the local dielectric environment and hence the resonance response but with increasing the complexity of the design and cost of fabrication [11]. On the other hand, many materials undergo a change of their EM properties as a function of temperature such as refractive index. Therefore, the metamaterials can be reconfigured thermally by changing the response to external temperature [12]. However, a cold medium is a required for any device, thus this approach is not practical for real applications.

Over the past several years, electrically reconfigurable metamaterials have drawn significant interest due to their ability to modify the EM properties of metamaterials. In this method, active devices such as varactor diodes, PIN diodes and micro-electro-mechanical systems (MEMS) have been used to achieve reconfigurability. A varactor diode is extensively studied in the literature [13-15]. It is modeled as a variable capacitance and this property brings various advantages such as simple integration and bias distribution due to using only one diode to achieve the tuning. However, low sensitivity to capacitance variation especially at high frequencies is the main drawback of this method besides the lack of suitable varactor diodes in terms of operating frequency and physical size [16]. The best switch in terms of high efficiency, low insertion loss and high isolation is MEMS, but this method has its own drawbacks such as high operation voltages (70-150V), complex fabrication requirements, expensive, and slow switching speed [16], [17]. On the other hand, the PIN diode is modeled as a variable resistance to represent the impedance of diode for varying bias voltage. The use of PIN diodes [18-20] in a metamaterials shares similarities with the use of varactors in terms of their applications, ease of integration, simple control, and fast switching. Moreover, the PIN diode is available for high frequency applications.

In this paper, we proposed and simulated a new metamaterial structure with low loss operates at $28 \mathrm{GHz}$, subsequently, the new structure is reconfigured using the copper strip as PIN diode formed in the gaps of the structure. Six frequencies, F1 to F6 are created with different negative refractive index values according to change the states of the switches for antenna beam steering in $5 \mathrm{G}$ mobile network applications.

\section{DESIGN AND NUMERICAL RESULTS OF THE PROPOSED METAMATERIAL UNIT CELL}

\subsection{Metamaterial Unit Cell Structure}

Bridge-shaped resonator (BSR) structure in the form of unit cell is depicted in the Figure 1. It consists of bridge shaped in the front face [see Figure 1(a)] and wire strip in the back face [see Figure 1(b)] and the both faces are connected through via in the center of the shape. The loop of square edge of the unit cell introduces inductance and gaps introduce capacitance thereby allows for the control of the resonant characteristic of the structure. BSR unit cell is printed on the $0.254 \mathrm{~mm}$ Rogers RT5880 thickness with relative permittivity 2.2 and tangent-loss of 0.0009 . The specifications of the design are tabulated in Table 1 . The material of the metallic BSR is lossy metal copper with an electric conductivity of $5.8 \times 10^{\wedge} 7 \mathrm{~S} / \mathrm{m}$ and the thickness of $0.035 \mathrm{~mm}$.

The ports and boundary conditions are assigned to excite the electromagnetics (EM) wave and extract effective constitutive parameters of the proposed unit-cell i.e. the refractive index. The $\mathrm{x}$ - and $\mathrm{y}$-axes are allocated with perfect magnetic conducting (PMC) and perfect electric conducting (PEC) boundary conditions, respectively. The two waveguide ports are assigned with open (add space) in the $\mathrm{z}$ direction as shown in Figure 1(c). The unit cell is simulated in the time domain using CST Microwave Studio, which is based on the finite integration technique.

Table 1. The BSR dimensions at $28 \mathrm{GHz}$

\begin{tabular}{cccc}
\hline Parameter & Value $(\mathrm{mm})$ & Parameter & Value $(\mathrm{mm})$ \\
\hline $\mathrm{X}$ & 3.7 & $\mathrm{U}$ & 0.27 \\
$\mathrm{Y}$ & 3.7 & $\mathrm{R}$ & 0.15 \\
$\mathrm{X} 1$ & 3.5 & $\mathrm{G}$ & 0.35 \\
$\mathrm{Y} 1$ & 3.5 & $\mathrm{H}$ & 0.254 \\
\hline
\end{tabular}




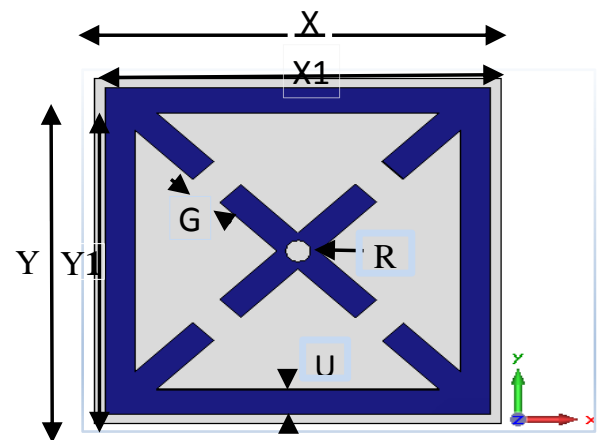

(a)

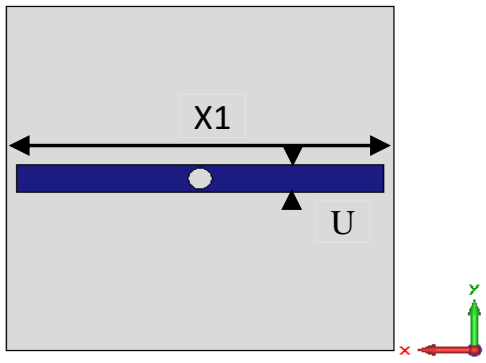

(b)

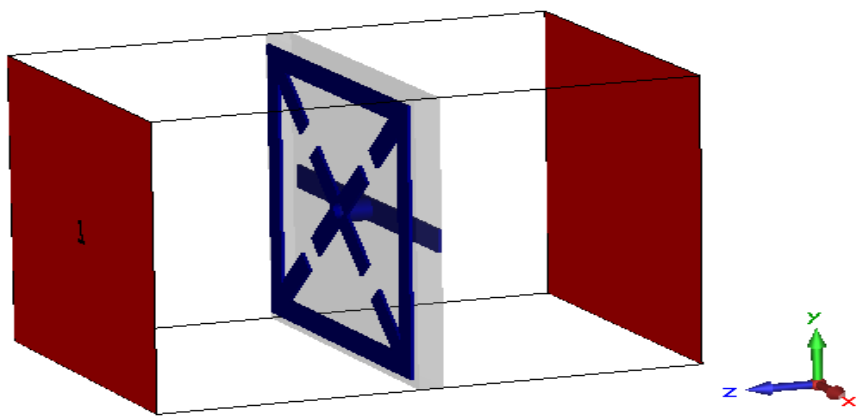

(c)

Figure 1. The BSR unit cell, (a) front face, (b) back face, and (c) the simulation geometry

\subsection{Low Loss Metamaterial}

In this section, the performance of the new metamaterial structure operates at MMW frequency range is presented. BSR unit cell is proposed to operate at $28 \mathrm{GHz}$ band which is the candidate band for $5 \mathrm{G}$ mobile network applications. Figure 2 reveals the reflection coefficient, $S_{11}$, of the proposed unit cell. It can be observed that the unit cell achieves a bandwidth of $1.53 \mathrm{GHz}$ at $-10 \mathrm{~dB}$. Noted, the main drawbacks inherent in the metamaterials are the narrow bandwidth and losses that limit their applications and late enable metamaterials based devices. In this work, the bandwidth of $1.53 \mathrm{GHz}$ is considered a good achievement despite the drawback of the metamaterial that presents a narrow bandwidth in nature. On the other hand, the losses in metamaterials at MMW frequency range are still a big issue and the quest for a low-loss structure at this range is highly demanded.

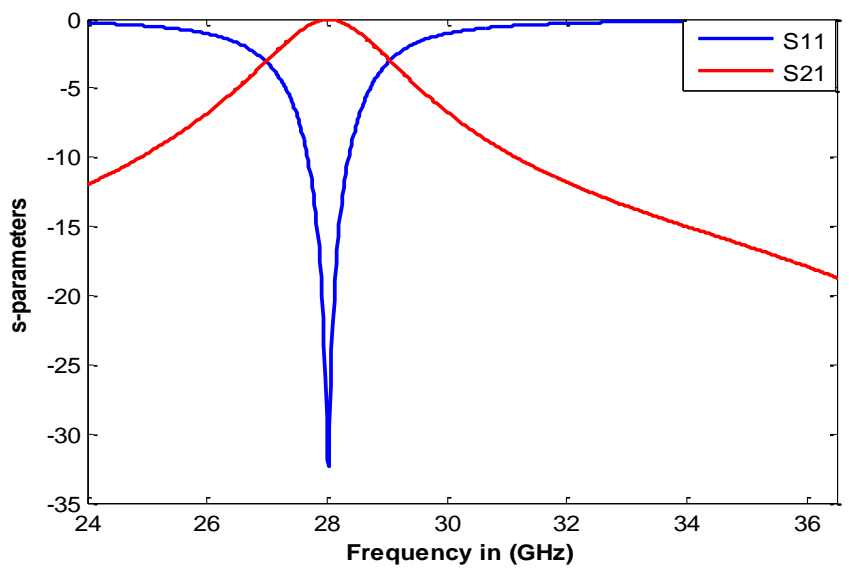

Figure 2. S-parameters of the proposed BSR structure 
In this work, the transmission coefficient is used to measure the loss of the metamaterial structure. For low loss, the peak of the transmission coefficient should be near zero $\mathrm{dB}$ in ( $\mathrm{dB}$ scale) or close to one in (linear scale) at desired frequency. The transmission band of the proposed BSR unit cell structure at $28 \mathrm{GHz}$ is shown in Figure 2. BSR shows the lowest loss by about $-0.06 \mathrm{~dB}(0.99$ in linear scale) at $28 \mathrm{GHz}$ in compression with recent literature [21]. In other words, BSR unit cell achieves near zero loss which almost achieves the full transmission at $28 \mathrm{GHz}$ MMW frequency.

The losses can give negative influences and adverse effects toward the realizations of the unique electromagnetic properties of the metamaterials. In other words, the double negative nature of the metamaterial should be investigated to prove the principle of the metamaterials and the influence of the losses. The Nicolson Ross Weir (NRW) approach based complex reflection and transmission is used to reconstruct the constitutive parameters [22]. In this method, the both effective permittivity and permeability are directly calculated from complex reflection and transmission coefficients then the refractive index can be calculated as follows:

$$
n=\sqrt{\varepsilon_{r} \mu_{r}}
$$

Figure 3 shows the real parts of the refractive index. The double negative nature of the BSR unit cell is verified here by the negative reflective index $n$.

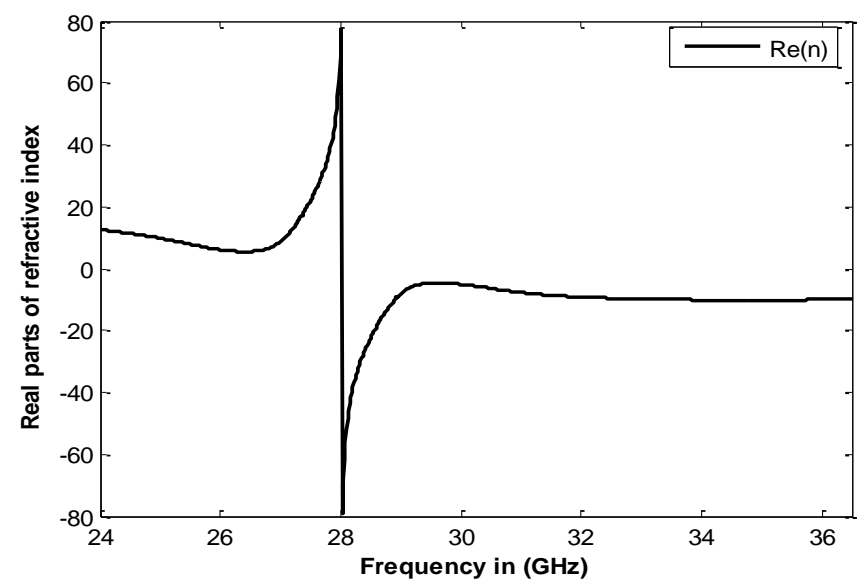

Figure 3. The real part of the effective refractive index

\section{DESIGN AND NUMERICAL RESULTS OF THE PROPOSED RECONFIGURABLE METAMATERIAL}

Based on the BSR structure in section 2, the reconfigurability property is added to the proposed metamaterial structure using copper strips act as PIN diodes as revealed in Figure 4. Four switches (D1, D2, D3, and D4) are inserted in the gaps of the two cross columns of the structure. In the simulation, we use a strip of copper to mimic the dimension of the PIN diode.

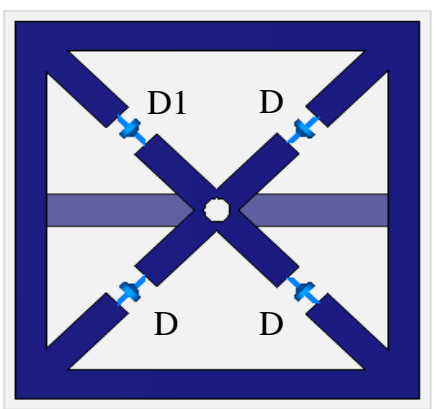

Figure 4. Reconfigurable metamaterial structure using PIN diodes 
The CST software is used to simulate the BSR with an active device, PIN diode, by representing the PIN diode as a copper strip. The electric, magnetic, and feeding open boundary conditions are assigned as in section 2.1. In the simulation, copper strip represents switch is ON while vacuum represents OFF state of the switch. The presence of the switches in the gaps of the proposed unit cell gives different values for resonant frequencies from F2 to F6 and also different values of the negative refractive index. The simulated reflection coefficient of the BSR unit cell with different states of the switches is depicted in Figure 5. It is noticeable that the resonance frequency is increased by inserting more switches in the gaps. In other words, the resonance frequency is increased when the values of capacitances (gaps) are decreased. The switches arrangement, resonant frequency and refractive index values of the proposed unit cell are tabulated in Table 2 .

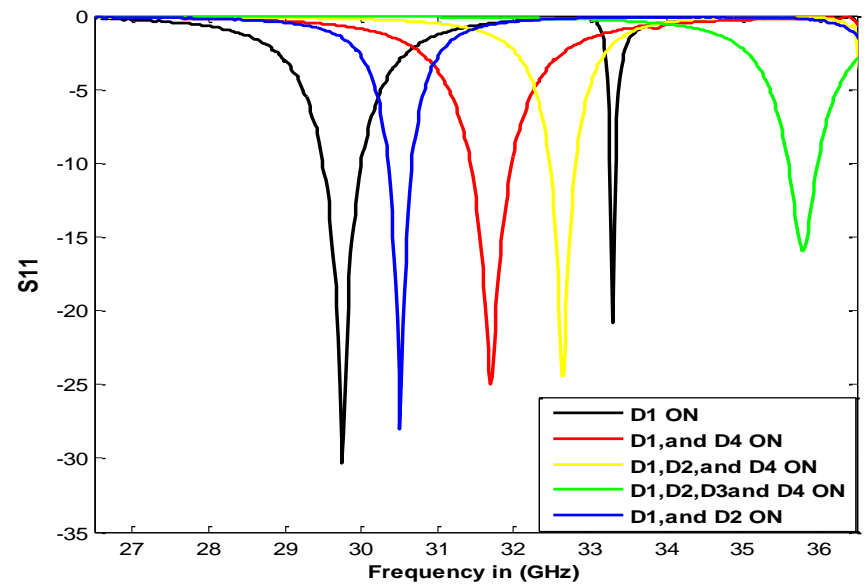

Figure 5. The reflection coefficients of the reconfigurable metamaterial structure

Table 2. Switch arrangement, resonant frequency and refractive index values of the proposed unit cell

\begin{tabular}{|c|c|c|c|c|c|c|c|}
\hline Figure No. & Frequency & D1 & D2 & D3 & D4 & $\begin{array}{l}\text { S11 of the BSR } \\
\text { unit cell }(\mathrm{GHz})\end{array}$ & $\begin{array}{l}\text { Refractive } \\
\text { index }(n)\end{array}$ \\
\hline Figures 2 and 3 & F1 & OFF & OFF & OFF & OFF & 28 & -80 \\
\hline \multirow{5}{*}{ Figures 5 and 7} & F2 & $\mathrm{ON}$ & OFF & OFF & OFF & 29.7 & -73 \\
\hline & F3 & $\mathrm{ON}$ & ON & OFF & OFF & 30.5 & -72 \\
\hline & $\mathrm{F} 4$ & ON & OFF & OFF & $\mathrm{ON}$ & 31.8 & -60 \\
\hline & F5 & $\mathrm{ON}$ & ON & OFF & $\mathrm{ON}$ & 32.7 & -55 \\
\hline & F6 & $\mathrm{ON}$ & ON & $\mathrm{ON}$ & $\mathrm{ON}$ & 36 & -45 \\
\hline
\end{tabular}

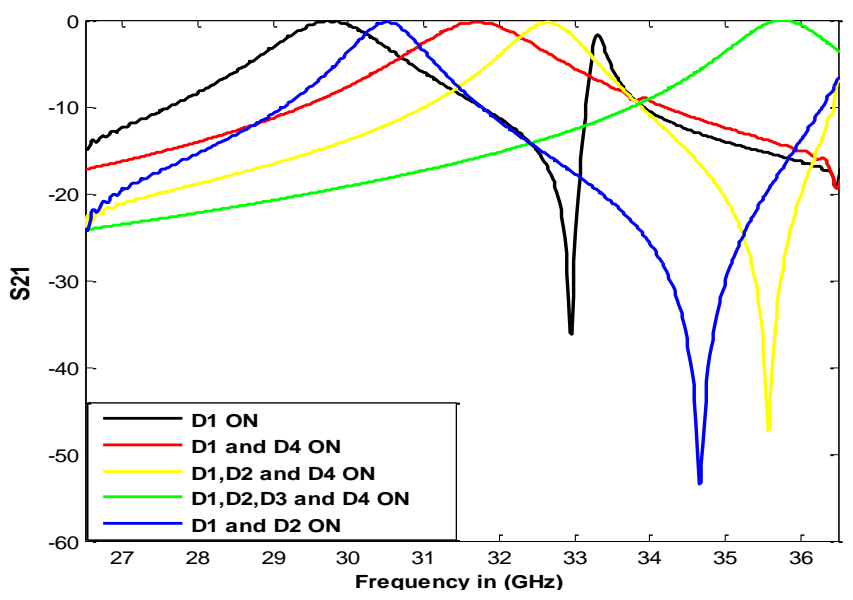

Figure 6. The transmission coefficients of the tunable metamaterial structure 
The transmission coefficients of the reconfigurable metamaterial structure are shown in Figure 6. The BSR unit cell achieves a low loss at all simulated states, thereby the unique EM properties of the metamaterial unit cell can be achieved. As mention in section 2, the NRW algorithm is used to retrieve the constitutive parameters of the BSR structure. Figure 7 presents real parts of the negative refractive index at each reconfigured frequency. It can be seen that the values of $n$ are altered according to the states of the switches. The lowest refractive index is $n=-80$ at F1 arrangement while $n$ goes to the highest $n=-45$ at F6 arrangement. These different values of $n$ can be exploited to steer the main beam of the $5 \mathrm{G}$ antenna with benefits over the conventional methods such as low profile structure, ease of integration on same antenna substrate, and the gain is enhanced through the switching process.

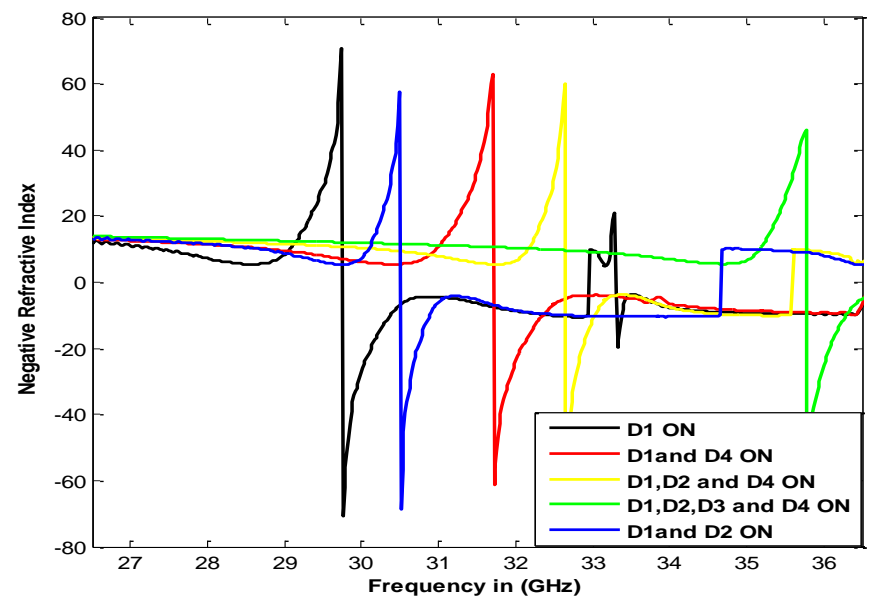

Figure 7. The real part of the negative refractive index of the tunable metamaterial structure

\section{CONCLUSION}

This paper presents a new BSR metamaterial unit cell operates at $28 \mathrm{GHz}$ for beam switching applications in 5G technology. The BSR introduces the lowest loss $(-0.06 \mathrm{~dB}(0.99$ in linear scale $))$ reported in the literature so far. Then the reconfigurable BSR unit cell is achieved using four switches positioned in the gaps of the structure. By controlling the ON and OFF states of the four switches, six different frequencies and refractive index values are created. The reflection and transmission coefficients and real parts of the effective refractive index at each reconfigured frequency are studied and investigated. Simulation results show that a high transmission and reflection peak occurs at each reconfigurable frequency.

\section{ACKNOWLEDGEMENTS}

This work was supported by Ministry of Higher Education (MOHE), Faculty of Engineering Technology, Research Center of Applied Electromagnetics, Research Management Centre, Universiti Tun Hussein Onn Malaysia (UTHM) under Grant (Vote No: GPPS U735 / FRGS 1614).

\section{REFERENCES}

[1] C. Sabah, "Realization of polarization-angle-independent fishnet-based waveguide metamaterial comprised of octagon shaped resonators with sensor and absorber applications," Journal of Materials Science. Materials in Electronics, vol. 27(5), pp.4777-4787, May 2016.

[2] V. G. Veselago, "The electrodynamics of substances with simultaneously negative values of and $\mu$," Soviet physics uspekhi, vol. 10(4), p.509, 1968.

[3] JB. Pendry, et al., "Low frequency plasmons in thin-wire structures", Journal of Physics: Condensed Matter, vol. 10(22):4785, Jun 1998

[4] A. Archambault, et al., "Superlens in the time domain," Physical review letters. 30, vol. 109(9), 097405, Aug 2012.

[5] Soric JC, et al., "Demonstration of an ultralow profile cloak for scattering suppression of a finite-length rod in free space," New journal of Physics. 25, vol. 15(3), 033037, Mar 2013.

[6] R. Rajni, et al., "Metamaterial Inspired Patch Antenna for ISM Band by Adding Single-Layer Complementary Split Ring Resonators," International Journal of Electrical and Computer Engineering (IJECE). 1 vol. 5(6), 1328-35, Dec 2015.

[7] L. Zhu, et al., "Low-loss magnetic metamaterial at $\mathrm{THz}$ frequencies by suppressing radiation losses," IEEE Transactions on Terahertz Science and Technology. vol. 13(6), pp. 805-11, Nov 2013. 
[8] Y. Fan, et al., "Low-loss and high-Q planar metamaterial with toroidal moment," Physical Review B. 15, vol. 87(11), p. 115417, Mar 2013.

[9] KL. Tsakmakidis, et al., "Negative-permeability electromagnetically induced transparent and magnetically active metamaterials," Physical Review B. 27 vol. 81(19), p.195128, May 2010

[10] AD. Boardman, et al., "Active and tunable metamaterials," Laser \& Photonics Reviews, 8 vol. 5(2), pp. 287-307, Mar 2011

[11] K. Fan and WJ. Padilla, "Dynamic electromagnetic metamaterials," Materials Today. 28 vol. 18(1),pp.39-50, Feb 2015.

[12] X. Duan, et al., "Dynamically tunable plasmonically induced transparency by planar hybrid metamaterial", Optics letters, vol. 38(4), p. 483-5, Feb 2013.

[13] J. Zhu, et al., "Tunable microwave metamaterial absorbers using varactor-loaded split loops," EPL (Europhysics Letters). vol. 112(5),54002. Dec 2015.

[14] T. Nesimoglu and C. Sabah, "A tunable metamaterial resonator using varactor diodes to facilitate the design of reconfigurable microwave circuits, " IEEE Transactions on Circuits and Systems II: Express Briefs, vol. 63(1),pp. 89-93, Jan 2016.

[15] J. Zhao, et al., "A tunable metamaterial absorber using varactor diodes", New Journal of Physics, vol. 15(4), 043049, Apr 2013.

[16] JP. Turpin JP, et al., "Reconfigurable and tunable metamaterials: a review of the theory and applications," International Journal of Antennas and Propagation. Vol. 22, May 2014.

[17] CP. Ho, et al., " Electrothermally actuated microelectromechanical systems based omega-ring terahertz metamaterial with polarization dependent characteristics," Applied Physics Letters. Vol. 104(16), 161104, Apr 2014.

[18] Razak A, et al., "Frequency Reconfigurable Epsilon Negative Metamaterial Antenna," International Journal of Electrical and Computer Engineering (IJECE). Vol. 7(3), Jun 2017.

[19] B. Zarghooni and TA. Denidni TA, "New fractal metamaterial unit-cell for microwave applications", InAntennas and Propagation (EuCAP), 2014 8th European Conference on 2014 Apr 6 ,pp. 978-979.

[20] R. Amiri, et al., "Reconfigurable metamaterial unit-cell with controllable refractive index," In Antenna Technology and Applied Electromagnetics (ANTEM), 2016 17th International Symposium on 2016 Jul 10, pp. 1-2.

[21] S Sonkusale, et al., "Terahertz metamaterials for modulation and detection," InSPIE Sensing Technology+ Applications, International Society for Optics and Photonics. pp. 948306-948306, May 2015.

[22] Z. He and Y. "Geng A wideband low-loss and small size left-handed metamaterial," InCommunication Technology (ICCT), 2015 IEEE 16th International Conference on 2015 Oct 18 , pp. 667-669.

[23] O. Luukkonen, et al., "A stepwise Nicolson-Ross-Weir-based material parameter extraction method", IEEE antennas and wireless propagation letters, vol. 10:1295-8., 2011.

\section{BIOGRAPHIES OF AUTHORS}

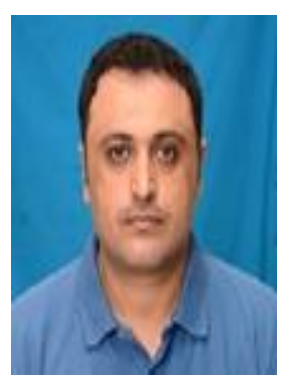

Bashar Ali Esmail received the B Eng. degree in Electrical Engineering (Telecommunications) with honours from Ibb University-Yemen, in 2008. He then obtained his M.Eng (Electrical Engineering) in 2016, at Universiti Tun Hussein Onn Malaysia. He is currently a on going PhD in Electrical Engineering at Electrical Engineering Technolgy Department, Faculty of Engineering Technolgy Universiti Tun Hussein Onn Malaysia. His research interest includes the areas of design of MTM unit cells, millimeter wave antenna and pattern reconfigurable MTM antenna.

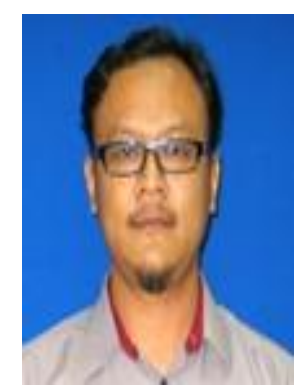

Huda A Majid received the B Eng. degree in Electrical Engineering (Telecommunication) from Universiti Teknologi Malaysia, in 2007. He then obtained his M.Eng in 2010 and PhD degrees in Electrical Engineering in 2013, at Universiti Teknologi Malaysia. He is currently a lecturer in the Department of Electrical Engineering Technology, Faculty of Engineering Technology, Universiti Tun Hussein Onn Malaysia. His research interest includes the areas of design of microstrip antennas, small antennas, Reconfigurable antennas, metamaterials structure, metalaterial antennas and millimeter wave antennas. He has published over 50 articles in journals and conference papers 

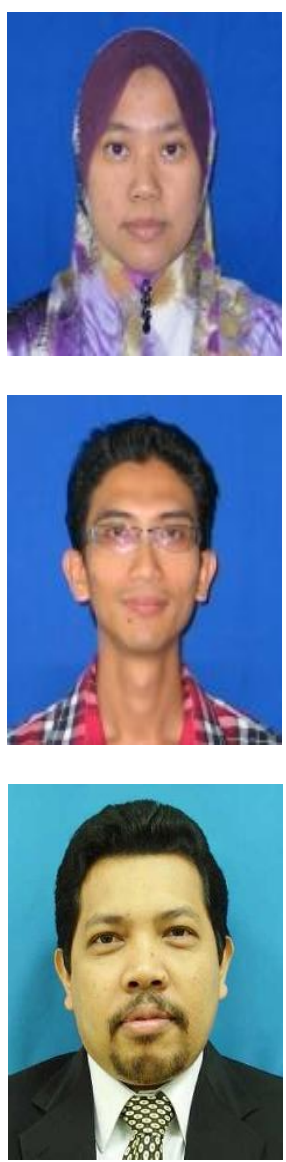

Zuhairiah Zainal Abidin was born in Kuala Lumpur, Malaysia, in 1978. She received the B.Eng.from the Universiti Teknologi Malaysia, in 2001, the M. Eng from the Kolej Universiti Tun Hussein Onn Malaysia, Johor, Malaysia, in 2003, and Ph.D. degree from Bradford University, U.K in 2011. Currently, she was a Senior Lecturer at Universiti Tun Hussein Onn Malaysia. Her current research interests include MIMO antenna design, electromagnetic bandgap (EBG) for wireless and mobile systems and high speed digital circuits and wearable antennas.

Samsul Haimi Bin Dahlan received the Bachelor's degree in Engineering from the Universiti Kebangsaan of Malaysia, Bangi, Malaysia, in 1999, the Master's degree in engineering from Universiti Teknologi Malaysia, Johor Bahru, Malaysia, in 2005, and the Ph.D. degree from Universite' de Rennes 1, Rennes, France, in 2012. He is currently the Head of the Research Center for Applied Electromagnetics, Universiti Tun Hussein Onn Malaysia (UTHM), Batu Pahat, Malaysia. His research interests include EMC, electromagnetic shielding, bioelectromagnetics, microwave devices, advanced antenna design, material characterization, and computational electromagnetics. Dr. Samsul has published many conference proceedings as well as journal papers in local and international journals.

Mohamad Kamal A Rahim received the B Eng. degree in Electrical and Electronic Engineering from University of Strathclyde, UK, in 1987. In 1989, he joined the Department of Communication Engineering, Faculty of Electrical Engineering Universiti Teknologi Malaysia Kuala Lumpur as an Assistant Lecturer A. He obtained his M.Eng Science from University of New South Wales Australia in 1992 and PhD degrees in Electrical Engineering from University of Birmingham UK in 2003. He is the Professor in RF and Antenna at Faculty of Electrical Engineering Universiti Teknologi Malaysia. His research interest includes the areas of design of dielectric resonator antennas, microstrip antennas, small antennas, microwave sensors, RFID antennas for readers and tags, Multi-function antennas, microwave cicuits, EBG, artificial magnetic conductors, metamaterials, phased array antennas, computer aided design for antennas and design of millimeter frequency antennas. He has published over 200 articles in journals and conference papers. 